\title{
A Study on HL7 Standard Message for Healthcare System Based on ISO/IEEE 11073
}

\author{
Am-Suk Oh \\ Dept. of Media Engineering, Tongmyong University, Busan, Korea \\ asoh@tu.ac.kr
}

\begin{abstract}
We studied the standard of HL7 message for healthcare system which can support diagnosis for healthcare provider and user. We studied the connection of standard medical devices, i.e., continua health alliance under the Android 4.x Bluetooth HDP (Health Device Profile) environments based on ISO/IEEE 11073 which is standard of information exchange among health device agent and service. And also we studied smart healthcare system using smart phone depending upon current trend. This system was studied on the environment of ISO/IEEE 11073 and HL7 standard.
\end{abstract}

Keywords: HL7standard message, Healthcare System, ISO/IEEE 11073

\section{Introduction}

U-healthcare technology can be used for diagnosis and medical treatment at any time, any place connected with networks for the aged and patients. It is expected that the Uhealthcare can solve the medical cost problem thru managing the chronic disease. [1] Rapid development of information and communication technology also supports their health concern via remote diagnosis, emergency medical treatment, self-monitoring and so on. Thus the communication system and interface system are considered important elements. [2]

However, each individual healthcare device contains unique protocol and related software of each different company and those protocols are not open to public. [3] Thus, there are a few data compatibility among such devices and the standardization to solve the problem became a very important issue. Nevertheless, the standardization is hardly done as increasing cost of the measuring- instruments and software. [4]

The object of smart healthcare service is focused on preventive healthcare and wellness. It also aims for the user oriented active service. This smart healthcare service is provided by smart phone application program. The total number of healthcare application program already exceeds 15,000 in May 2011. [5] Currently, smart healthcare service can support various services like basal physical fitness, caloric intake, checking heartbeat and so on. [6]

For the interface with healthcare diagnosis system, it should support communication function of the health, feedback information which is also provided by healthcare server/gateway. We tried to study smart gateway which can support the interface of medical devices and the contents thru information transformation module based on HL7, Android 4.x Bluetooth HDP standard [7].

\section{Healthcare System}

A Current existing home and mobile healthcare system is shown in Figure 1. 


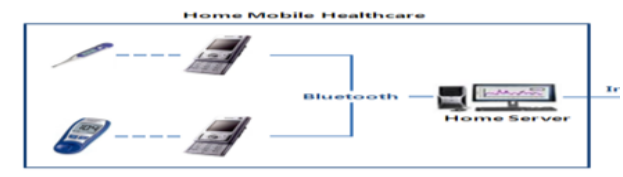

Figure 1. Configuration of Existing Home and Mobile Healthcare System

Via home server it monitors data which come from a smart phone linked with special medical devices. This home \& healthcare system is dependent to the medical devices linked with a smart phone. Thus, the interconnection among medical devices and healthcare service is so limited.

Recently, home healthcare system support ISO/IEEE11073 PHD, HL7 standard. It also supports medical information exchange by unlimited connection for the standard healthcare devices under HL7 environment. This concept is shown in Figure 2.

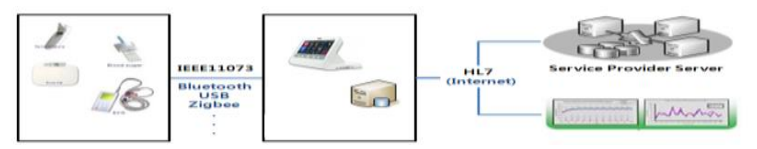

Figure 2. Standard Home Healthcare System

However, this kind of home healthcare system can't blend with smart application s like health and medical related services. It can't solve location dependent problems of gateway although it supports the monitoring interface of a smart phone. Without gateway it can't connect to the smart phone.

\section{HL7 Message}

\subsection{HL7 Standard}

HL7 is a set of rules which inter-operate among different kinds of information for medical application and this standardization is developed by stakeholders like a user, a supplier and other medical information concerned members because they wanted every different type of application service to be treated and satisfied to the requirements. Thus, it can transfer information among organizations concerned and the doctor who treats the patients can get the information for diagnosis and treatment. The route is set up via network and trigger event for the information interchange. Figure 3 shows the structure.

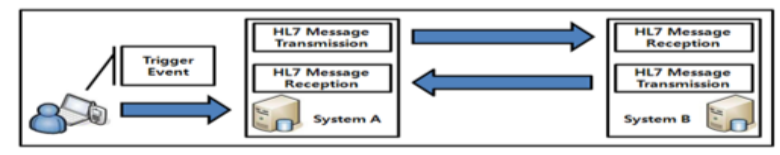

Figure 3. Structure of HL7

Data is transmitted from one system to the other system as a type of message and vice versa. After that action, one trigger event will be finished.

HL7 specifies following 3 events.

- $\quad$ abstracting message definition: structure of message

- $\quad$ encoding rules: message expression for transmission

- $\quad$ triggering events: application event for message exchange 


\subsection{HL7 Message Structure}

Standard of HL7 is composed of messages, segments, fields and data type. A message has several segments, and a segment has several fields. The relationship between fields and data type which include itself is one to one structure.

3.2.1. Message: Message is the smallest unit of data transfer and has segments. Each message has message structure suitable for its own purpose. MSH (Message Header) segment is the first place of all the segments.

- $\quad$ Message is started by MSH

- $\quad$ Message is identified by message type and trigger event

- $\quad$ Message type and trigger event has one too many relationships

- $\quad$ All message types and trigger evens starting with 'Z' are used as a user defined message.

3.2.2. Segment: Segment is a logical group of fields and it is divided into default segment and optional segment. Default segment means essential segment in message and optional can be used just one time or several times. Each segment has a unique name of 3 letters. For the representing segment into character string first field should be segment ID and each field value should be defined continuously. At the end, segment separator $\langle\mathrm{CR}\rangle$ is followed.

3.2.3. Field: Field is a set of components and components are structured as sub components. Contents of message are presented in the field of segment and it allows null value. Field has sequence number of internal segment. Maximum length attribute of field defines maximum number of characters and it can be adjusted by characteristics of each domain. The maximum length includes component and sub component and repetition field by repetition identifier should be calculated differently because maximum length is used for the length of no duplication. Field can be repeated like segment and it is divided into 3 types such as no repetition, continuous repetition and limited repetition depending upon the conditions.

3.2.4. Data Type: Data type supports contents of data fields and it includes multi components or sub components. Generally, HL7 does not limit the length in data type. Data type is composed of composite which is the set of different types of data and primitives which is the set of same data type.

\subsection{Implementation of UHI Message}

We implemented the message for health care system based on ORI (Observation Reporting Interface) which is the standard of medical data communication between medical devices and HL7 protocol. The main issue of this ORI standard is the interchange of the data, object and model defined on ISO 11073 to HL7 protocol using HL7 coding rules and XML form.

This standard supports 3 types of message profile.

- $\quad$ ORU^R30: Order not supplied Create message

- $\quad$ ORU^R31: Order not supplied Search message

- $\quad$ ORU^R32: Order supplied message

We added PFI, PCI segments designed to the standard HL7 ORU^RXX structure and its structure coincide with existing standard. Thus, we designed and implemented that ISO 11073 protocol can be applied on standard health care system or even non-standard medical devices. Figure 4 and 5 shows the implementation result of $\mathrm{UHI}^{\wedge} \mathrm{U} 01, \mathrm{UHI}^{\wedge} \mathrm{U} 02$ 
message based on ORU^RXX message structure and inside contents of red line is the PFI, PCI segments we added.

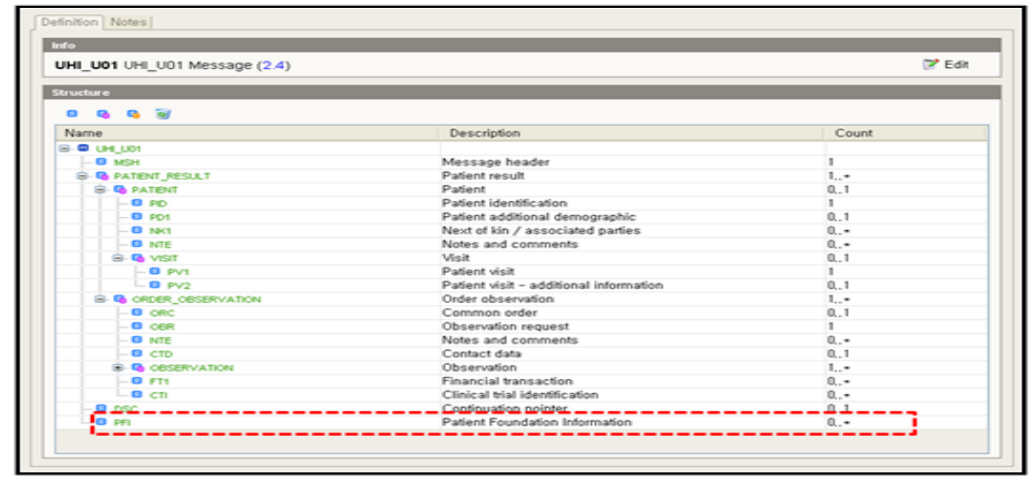

Figure 4. UHI^U01 Message Structure

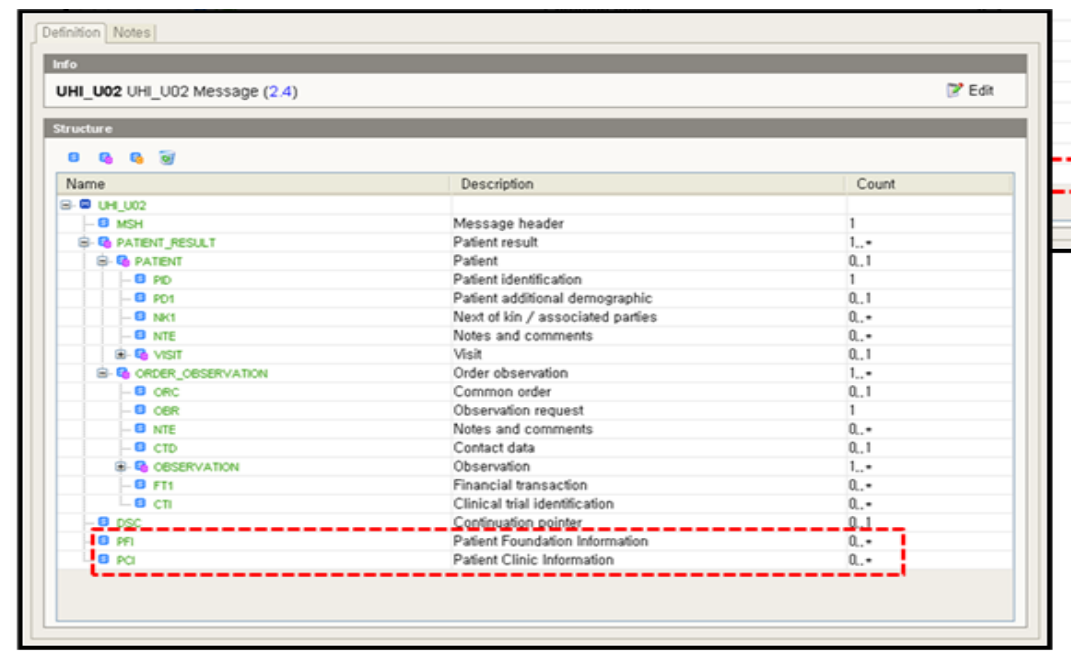

Figure 5. UHI^U02 Message Structure

\section{Smart Healthcare System}

Smart Healthcare System designed in this thesis is composed of Bluetooth standard PHD, and smart gateway, DSSH (Decision Support System for Home Healthcare) shown in Figure 6.

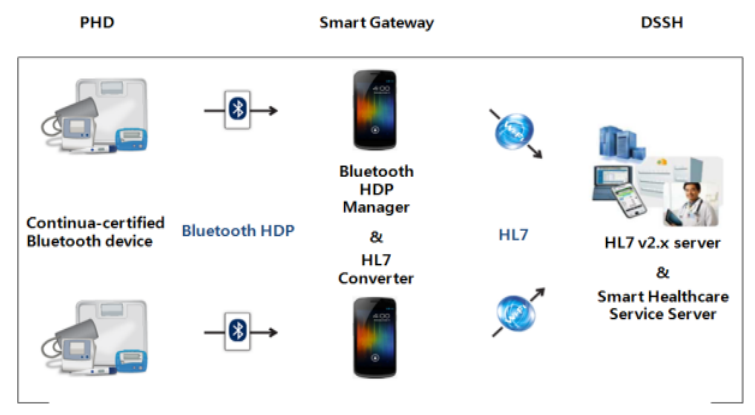

Figure 6. Structure of Smart Healthcare System 


\subsection{Smart Gateway}

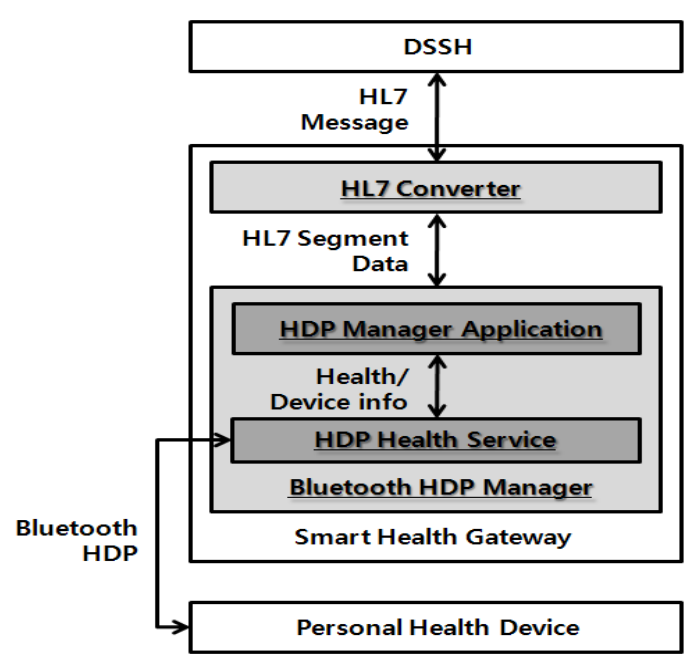

Figure 7. Smart Gateway Software Module

Smart gateway is information conversion gateway which gathers medical data from PHD and exchanges information based on HL7 standard. Software module of smart gateway is composed of Bluetooth HDP manager and HL7 standard message creation function, HL7 converter. They inter-connect with DSSH via HL7 message exchange and it is shown in Figure 7.

HDP health service module connects with PHD using Antidote stack API function proposed on this thesis. HDP Manager Application is an application program which provides Bluetooth HDP function to the user. HL7 converter converts IEEE 11073 into HL7 and vice versa. It also exchanges messages of work-list server in DSSH and HL7 v2.x Message.

\subsection{HL7 Converter and DSSH}

HL7 converter module is composed of various types of remote systems like medical treat system, healthcare management system and so on. We designed HL7 work-list server which exchanges HL7 standard message and Health Service Server which manages user health information in DSSH structure shown in Figure 8.

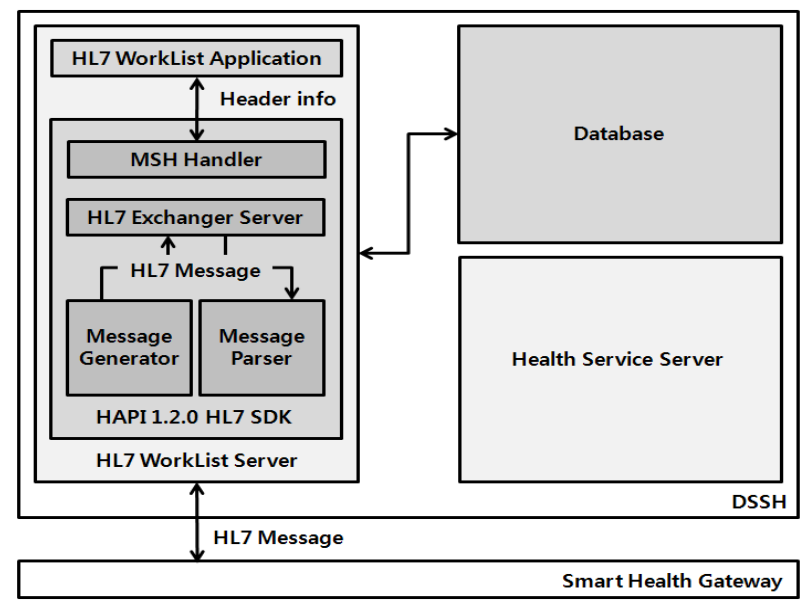

Figure 8. Structure of HL7 converter and DSSH 
HL7 work-list server is a socket communication server based on TCP/IP protocol. It confirms HL7 v2.x standard message from smart gateways and manages database. Message Generator creates a message and Message Parser parses received the message. MSH Handler classifies message type, sends it to a database and manages the message received from smart gateway

\section{Conclusions}

Healthcare system is utilized for the sharing of patient's clinic data among hospitals based on ISO/IEEE11073 and HL7 standard. We studied the standard of HL7 message for smart healthcare system which can support diagnosis for the healthcare provider and the user. Smart healthcare system supports to link existing U-healthcare system and HL7 standard. We defined segment field for remote diagnosis, live body monitoring, emergency medical treatment and designed HL7 infra-structure message depending on trigger event under the U-Health service environment. The design contents was implemented as the library of UHI type which is part of U-Health message and it is confirmed via virtual HIS database testing. And also healthcare service provided by smart phone application level could be linked with various types of service application. Thus adaptation of healthcare service becomes easier and extension could be expected more hopeful. We hope this study could contribute to the smart healthcare system.

\section{References}

[1] J. Escayola, M. M. nez-Espronceda, P. Mu oz, "Seamless Integration of ISO/IEEE11073 Personal Health Devices and ISO”, TELEMEDICINE JOURNAL AND E HEALTH, vol. 16, no. 10, (2010).

[2] D. P. Bogia, "ISO/IEEE 11073 Personal Health Devices Tutorial," IEEE, (2010).

[3] IEEE STD 11073-20601 Application Profile - Optimized Exchange Protocol, (2008).

[4] S. Koch, "Ubiquitous Carein Aging Societies-A SocialChallenge", Studiesin HealthTechnologyand Informatics, vol. 134, (2008), pp.89-95.

[5] K. S. Jeong, E. Y. Jung and D. K. Park, "Trend of Wireless u-Health," Proc. of the 9th International Symposium on Communications and Information Technology, (2009), pp.829-833.

[6] N. Watthanawisuth, T. Lomas, A. Wisitsoraat, A. Tuantranont, "Wireless Wearable Pulse Oximeter for Health Monitoring using Zigbee Wireless Sensor Network," Proc. of the International Conference on Electrical Engineering/Electronics Computer Telecommunications and Information Technology, pp. 575-579, (2010).

[7] A. Czabke, S. Marsch, T. C. Lueth, "Accelerometer Based Real-TimeActivity Analysisona Microcontroller", Proc. of the 5th International Conferenceon Pervasive Computing Technologiesfor Healthcare and Workshops, pp. 40-46, (2011).

[8] U. Varshney, "Wireless Medication Management System, Design and Performance Evaluation", Proc. of the Wireless Telecommunications Symposium, pp. 1-8, (2011).

[9] J. -H. Jeon, S. -I. Kang, G. -H. Kim, A. - S. Oh, J. -H. Lee and S. -W. Choi, "A Study on Home Healthcare System based on Personal Mobile Gateway", International Conference of KIICE 2012, vol. 5, no. 1, pp. 196-197, (2012).

[10] J. -H. Jeon, S. -I. Kang, G. -H. Kim, A. -S. Oh, S. -W. Choi and O. -H. Kwon, "A Study on Smart Gateway based on Android 4.x Bluetooth HDP(Health Device Profile)", Computers, Networks, Systems, and Industrial Applications CNSI 2012, vol. 8, no. 1, pp. 515-519, (2012).

\section{Authors}

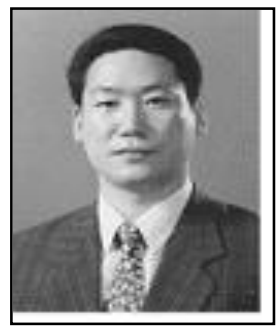

Am-Suk Oh, Received the B.S. and M.S. degrees in computer science from Busan National University and Chung-Ang University, respectively. He received $\mathrm{Ph}$. D at the computer engineering of Busan National University. He is currently with the Department of Media Engineering, Tongmyong University as a professor. His research interests are Database, Healthcare System and Medical Information System. 